\title{
Vetas epitermales y su relación con un posible depósito tipo IOCG en Cerro Colorado Chico, Ancash-Perú
}

\author{
Epithermal veins and their relationship with a possible IOCG type deposit in \\ Cerro Colorado Chico, Ancash-Peru
}

\author{
Jesús Alberto Torres Guerra ${ }^{1}$, John Franklin Cerrón Sarcco²
}

Recibido: 16/08/2019 - Aprobado: 07/042021 - Publicado: 18/06/2021

\begin{abstract}
RESUMEN
Este artículo pertenece al proyecto: "Estudio del magmatismo Mesozoico y sus implicancias en la determinación de un modelo de exploración geológico-estructural relacionado con la ocurrencia de Yacimientos tipo IOCG en la Cordillera de la costa y llanuras pre-andinas de los Andes Peruanos" elaborado dentro del convenio de INGEMMET y la UNMSM. Durante las actividades de campo se enfatizó la cartografía geológica de las zonas C-1 y C-2, identificando la presencia de vetas epitermales, cortando a la serie de afloramientos intrusivos del Batolito de la Costa

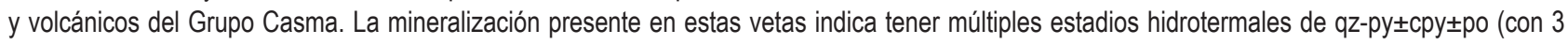
generaciones de qz), zonas de alteración argílica con una asociación mineralógica de sílice-arcillas (caolínita/dickita-illita)-sericita, además de trazas de alunita-pirofilita, gradando lateralmente a un zona de alteración hidrotermal compuesto por cuarzo- hematita-goethita-sericita-clorita (relacionado con evidencias superficiales de un depósito tipo IOCG oculto ubicado en la Zona C-2). Estas vetas presentan anomalías de Au, Cu, As y Zn, además de una correlación de Sc-V-P-Ti. Los resultados de los estudios de microtermometría en inclusiones fluidas bifásicas de las vetas muestran temperaturas de homogenización varían de 138 a $329^{\circ} \mathrm{C}$, y porcentajes de salinidad están desde 0.70 a $8.95 \%$ Equivalente en peso de $\mathrm{NaCl}$. Cabe mencionar que en una muestra estudiada proveniente de la zona C-1, se encontraron inclusiones fluidas trifásicas con temperaturas de fusión de hielo con un mínimo de $-5.8^{\circ} \mathrm{C}$ y un máximo de $-0.4^{\circ} \mathrm{C}$, temperaturas de homogenización que están entre 560 a $605^{\circ} \mathrm{C}$, y porcentajes de salinidad que van desde 44.82 a $47.66 \%$ Equivalente en peso de $\mathrm{NaCl}$. Dada las evidencias, se propone que estas vetas epitermales son de sulfuración intermedia ligadas a un Pórfido no aflorante ubicado en C-1, mientras que en C-2 se sugiere la existencia de un yacimiento IOCG oculto, donde posiblemente habría una relación espacio-temporal del IOCG y el pórfido, como detallan autores de otros artículos.
\end{abstract}

Palabras clave: Epitermales; IOCG; petromineralogía; geoquímica; estadística; inclusiones fluidas.

\begin{abstract}
This article belongs to the project: "Study of the Mesozoic magmatism and its implications in the determination of a model of geological-structural exploration related to the occurrence of IOCG deposits in the Cordillera of the coast and pre-Andean plains of the Peruvian Andes" developed between INGEMMET and UNMSM. During field activities, geological mapping of zones C1 and C-2 was emphasized, identifying the presence of epithermal veins, cutting the series of intrusive outcrops of the Coasthal Batholith and volcanic of the Casma Group. The mineralization presents in these veins indicates to have multiple hydrothermal stages of qz-py \pm cpy \pm po (with 3 generations of qz), with zones of argillic alteration with a silice-clays (kaolinite/dickite-illite)-sericite mineralogical association, in addition having traces of alunite and pyrophyllite, laterally grading a large zone of quartz-hematite-goethite-sericite-chlorite alteration (related to the surface evidence of some IOCG Type deposit, mostly in Zone C-2). These veins have anomalies of $\mathrm{Au}, \mathrm{Cu}, \mathrm{As}$ and $\mathrm{Zn}$, in addition to a correlation of Sc-V-P-Ti. The microthermometric results in biphasic fluid inclusions of the veins show homogenization temperatures between 138 to $329^{\circ} \mathrm{C}$, while the salinity ranges are 0.70 to $8.95 \% \mathrm{Eq}$. $\mathrm{NaCl}$ weight. It should be mentioned that in the sample of Zone $\mathrm{C}-1$, there are three-phase fluid inclusions with ice melting temperatures minimum $-5.8^{\circ} \mathrm{C}$ and maximum -0.4 ${ }^{\circ} \mathrm{C}$, homogenization between 560 to $605^{\circ} \mathrm{C}$, and salinity ranges vary from 44.82 to $47.66 \% \mathrm{Eq}$. NaCl weight. Given the evidence, it is proposed that these epithermal veins would be Intermediate Sulphuration linked to a non-emerging Porphyry in Zone C-1, while in C-2 the probable presence of a deep IOCG type deposit where there would be a probable relationship between both, as detailed by authors of other articles.
\end{abstract}

Keywords: Epithermal; IOCG; petromineralogy; geochemistry; statistic; fluid inclusions.

\footnotetext{
1 Docente de la Universidad Nacional Mayor de San Marcos, Facultad de Ingeniería Geológica, Minera, Metalúrgica y Geográfica. Lima, Perú. E-mail: albertorresg@hotmail.com - ORCID: https://orcig.org/0000-0001-8186-5249

2 Bachiller de la Universidad Nacional Mayor de San Marcos, Facultad de Ingeniería Geológica, Minera, Metalúrgica y Geográfica, E. P. Ingeniería Geológica. Lima, Perú. E-mail: jcs12160005@gmail.com - ORCID: https://orcig.org/0000-0003-1640-3800
} 


\section{INTRODUCCIÓN}

El "Estudio del magmatismo Mesozoico y sus implicancias en la determinación de un modelo de exploración geológico estructural relacionado con la ocurrencia de Yacimientos tipo IOCG en la Cordillera de la costa y llanuras pre-andinas de los Andes Peruanos" es un proyecto ejecutado entre la UNMSM e INGEMMET, el artículo presentado es un estudio detallado dentro del citado proyecto. Esta investigación comenzó usando en gabinete la teledetección que nos permitió localizar el prospecto Cerro Colorado Chico.

Como antecedentes, encontramos estudios realizados desde los años setenta por compañías mineras como Cerro de Pasco Corp., y Newmont Mining más recientemente, entre otras e instituciones como INGEMMET, etc. Con respecto a las actividades en el campo se enfatizó en dar un soporte integral apoyado en las particularidades morfológicas, estructurales y litológicas del área de estudio y se puso énfasis en la zona que alberga a las vetas epitermales, además de analizar una posible relación con un yacimiento tipo IOCG.

\subsection{Ubicación}

El área de estudio se localiza a $230 \mathrm{~km}$ al norte de Lima, en el anexo de Pararín, distrito de Paramonga, provincia de Huarmey, región Ancash (Figura 1). Es de fácil acceso siguiendo por la Panamericana Norte y luego tomando desvíos hacia el oeste por caminos carrozables.

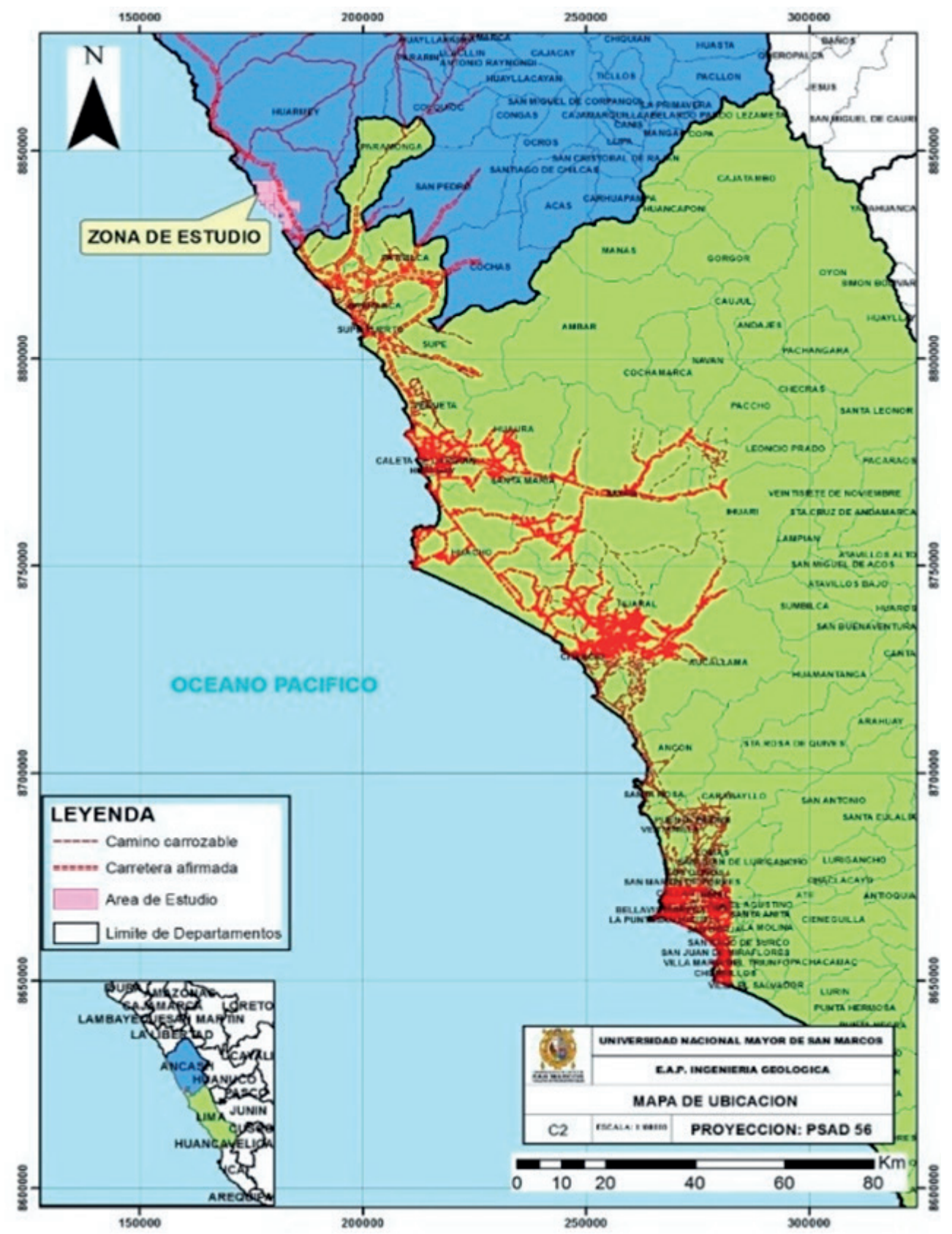

Figura 1. Plano de ubicación del área de estudio (Cerro Colorado Chico) (Datos: IGN) 


\subsection{Marco Geológico}

El área de investigación está ubicada dentro de la Faja Costanera del Norte del Perú (Figura 2), la cual la compone el Grupo Casma (Guevara, 1980) del Cretáceo inferior (130 a $105 \mathrm{Ma}$.), que genéticamente se relaciona con un ciclo volcánico submarino y subaéreo, generados por un volcanismo discontinuo que se dio lugar en una región volcánica marginal mientras actuaba el magmatismo del Arco Casma (Soler, 1990), mostrando su intercalación con secuencias volcánicas (tobas y derrames) y sedimentarias (arcillitas, areniscas, etc.) (Cobbing, 1973; Jacay, 2005). Este arco estuvo presente durante un periodo de subducción de débil impulso (según Mamani, Navarro, et al., 2010) generando así el plano de Benioff un ángulo de $60^{\circ}$ (Soler,
1990; Pierre Soler \& Bonhomme, 1990), caracterizando a este arco por pertenecer a las series toleiticas a calcoalcalinas (Cueva et al., 2010) hasta inclusive alcalinas (Mamani, Wörner, et al., 2010).

A finales del Cretáceo, el Grupo Casma es plegado dadas sus componentes volcánicas y sedimentarias (Cobbing, 1973) configurándose pliegues amplios con ejes axiales subverticales y subhorizontales de dirección andina y andina - normal según Guevara (1980) y que debido a su cercanía al borde ascendente, pasan a conformar zonas con intensa deformación (Faja Plegada Mesozoica) con un alto nivel de metamorfismo particularmente durante la fase de Orogenia Andina. Este plegamiento, por último, está cortado por fallas de diversas magnitudes.

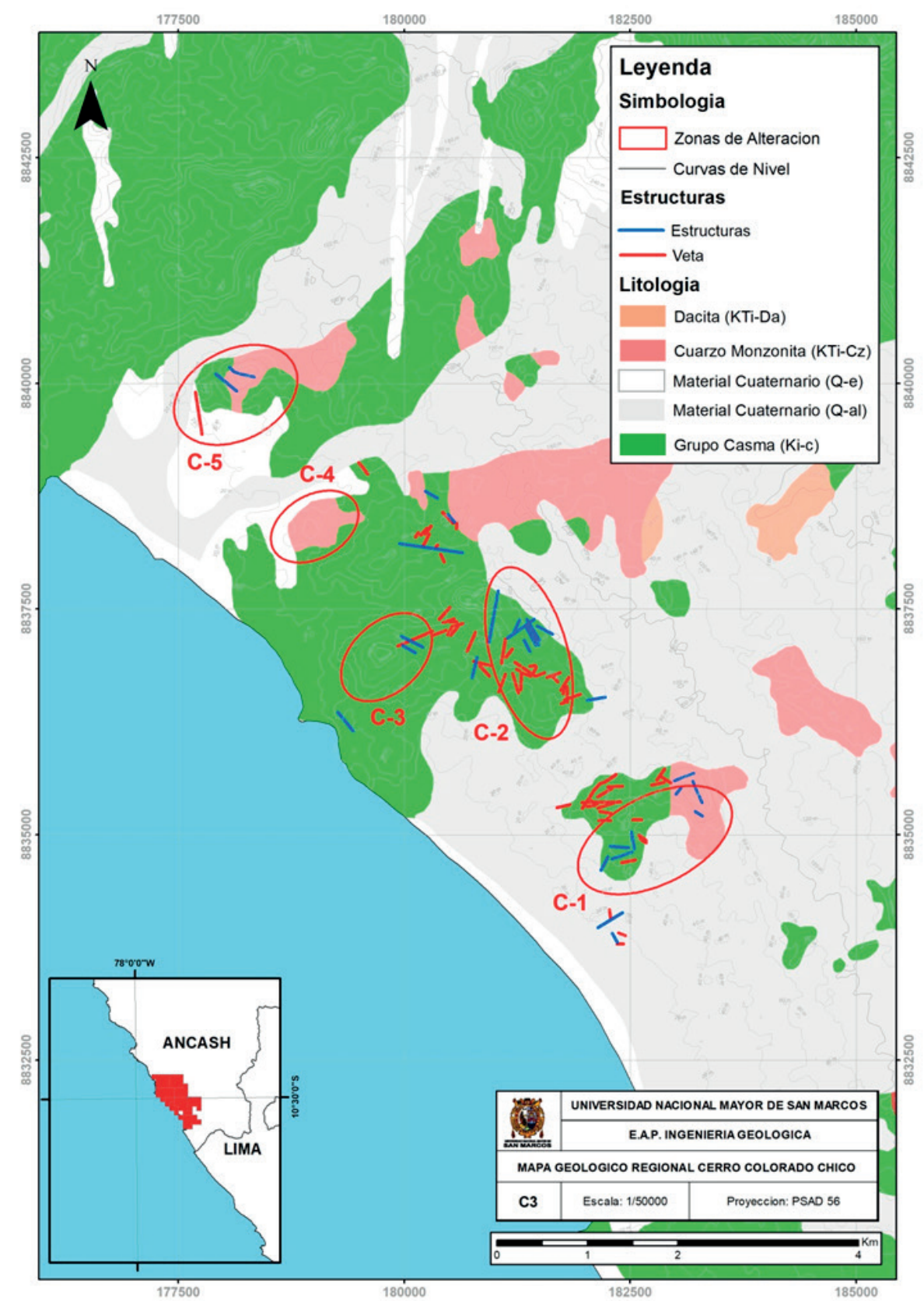

Figura 2. Plano Geológico Regional Cerro Colorado Chico 
Cabe mencionar que el Gpo. Casma fue intruido por intrusiones plutónicas y subvolcánicas conformadas por rocas alcalinas y ácidos separadas por eventos de oxidación (según Cobbing, 1973) enmarcados dentro del Batolito de la Costa, segmento Lima (Pitcher, 1979). Cabe mencionar que Jacay et al.(2005) señalan que las intrusiones poseen formas tabulares de grosor variado, con orientación noreste para los niveles inferiores, y nor-noreste para los niveles superiores. Soler (1990) y Soler \& Bonhomme (1990); indican que el desarrollo de una mayor velocidad de convergencia producido por el plano de Benioff con una inclinación de $45^{\circ}$, se evidencia con los múltiples eventos magmáticos que dieron lugar al Batolito de la costa. Por otro lado, este incremento en la velocidad nos ayuda a entender el cambio que hubo desde una cuenca de tipo volcánica marginal inicial a un arco de islas donde cuyo frente mientras sucedía el emplazamiento se desarrollaba hacia el este originando así su ampliación.

Por tal motivo, la secuencia de unidades estratigráfica del Grupo Casma, está conformada desde el piso hacia el techo por lavas almohadilladas de la Formación Punta Gramadal, sobreyaciendo la Formación La Zorra con tobas andesíticas, Formación Breas con cherts, Formación Lupin con derrames andesíticos almohadillados y piroclásticos de la Formación Pararin. Luego tenemos en discordancia erosiva al Grupo Calipuy y a la Formación Fortaleza. Por último, y al tope de la columna estratigráfica tenemos depósitos cuaternarios de variada tipología (aluviales, eluviales y eólicos (Cobbing, 1973; Guevara, 1980).

Cabe mencionar que se tiene certeza de la presencia de cuatro fases de deformación: la primera está relacionada con el levantamiento de la cordillera de los andes, la segunda con la Orogenia Andina que condiciono el plegamiento y fallamiento del Grupo Casma, la tercera que divide a los Andes en bloques fallados, y finalmente la cuarta relacionada con el tectonismo en bloques de los Andes entre el Plioceno y el Pleistoceno.

\section{METODOS}

La investigación realizada es descriptiva ya que permite obtener las características y propiedades de los fenómenos observados, utilizando para tal fin criterios de clasificación que permiten definir su estructura y composición. De acuerdo con la fuente de información es del tipo: documental y de campo. Además, es cuasi experimental y finalmente explicativa ya que busca descubrir los orígenes o las causas de un determinado fenómeno, con la finalidad de conocer las condiciones en las que ellos se producen, fundamentándose en bases teóricas.

La presente investigación se desarrolló en diferentes etapas, descritas a continuación:

Etapa 1: En esta etapa basada en la técnica documental, se realiza la búsqueda y recopilación de todas las fuentes bibliográficas relacionadas con estudios previos y los fundamentos teóricos de la mineralización del tipo IOCG y pórfidos. Para la parte de la geología regional se ha revisado diferentes estudios relacionados con la geología del segmento costero y central de los andes peruanos. En esta etapa se sugiere la estructura preliminar y específica de la investigación.

Etapa 2: Se descargan informes y mapas del INGEMMET, así como imágenes de sensores remotos como ASTER y Landsat 8 para realizar el análisis de las características litológicas, morfológicas, de alteraciones, mineralización y estructurales del contexto geológico regional.

Etapa 3: Mediante la técnica de campo, se realiza una cartografía geológica a escala 1:10 000 en función de la observación directa, lo que permite confrontar la teoría con la práctica, y luego correlacionar la información encontrada entre una y otra zona.

Etapa 4: Se realizan estudios al microscopio y de difracción de rayos $\mathrm{x}$ con el propósito de determinar de manera precisa las características litológicas y de alteraciones hidrotermales; también se hacen estudios de inclusiones fluidas para aplicar el diagrama de salinidad vs temperatura de homogenización y determinar el tipo de yacimiento.

Etapa 5: Análisis, interpretación de los resultados y redacción del informe.

\section{RESULTADOS}

\subsection{Alteración y Mineralización - vetas epitermales}

En el área de estudio, se diferenciaron 5 Zonas anómalas, donde las zonas C-1 y C-2 están localizadas en la Franja Metalogenética de yacimientos IOCG del Cretáceo.

En la zona denominada C-1, la mineralización epitermal está compuesta por estructuras tabulares de cuarzo gris (llegando a un máximo de 2 metros de ancho) con 3 tipos de qz, patinas de jarosita-hematita, mientras que en la Zona C-2 es donde presentan algunos minerales secundarios de $\mathrm{Cu}$ (malaquita y covelita). Estas vetas también cortan a los cuerpos intrusivos, con el nombre de campo "Stocks de Qz" que comprenderían a las rocas intrusivas dacitas porfiríticas alteradas.

Como resultado del estudio de microscopia hecho en las vetas, mostraron la presencia de inclusiones de cuarzo III en intersticios dentro de cuarzo II, con impregnaciones de arcillas y sericita en las cavidades, además de hematita y goethita supérgenas. En ocasiones existen trazas de minerales como carbonatos y rutilos, así como diseminaciones (¿selectivas?) de pirita anhedral a subhedral y calcopirita siendo incluidas en el cuarzo II. Además, existen brechas silíceas con 2 generaciones de cuarzo con diseminaciones de pirita con inclusiones de pirrotita (Figura 3).

El análisis estadístico de vetas utilizando información recopilada e información propia (siendo una estadística paramétrica) evidencio anomalías entre las que destacan del Au con un máximo de 3.39 ppm (Zona C-2), Cu con un máximo de 1030 ppm (Zona C-3), As llegando a un máximo de 380 ppm y Zn con un máximo de 786 ppm. Así mismo, el resultado de las correlaciones bivariadas muestra la correlación de Sc-P-V-Ti. 


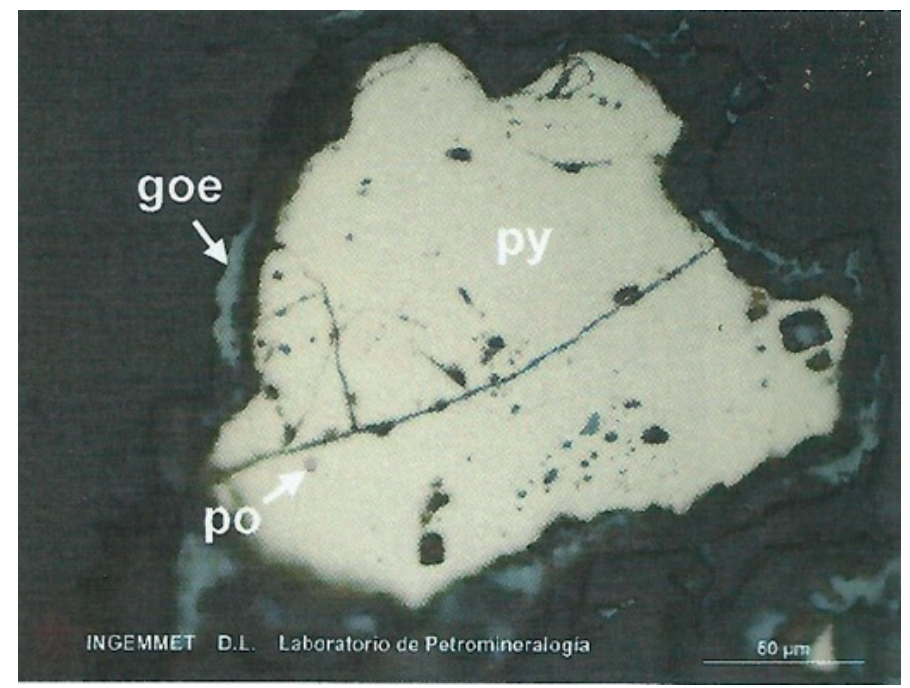

Figura 3. Fotomicrografías de sección apreciando a la pirita y trazas de pirrotita en brechas siliceas

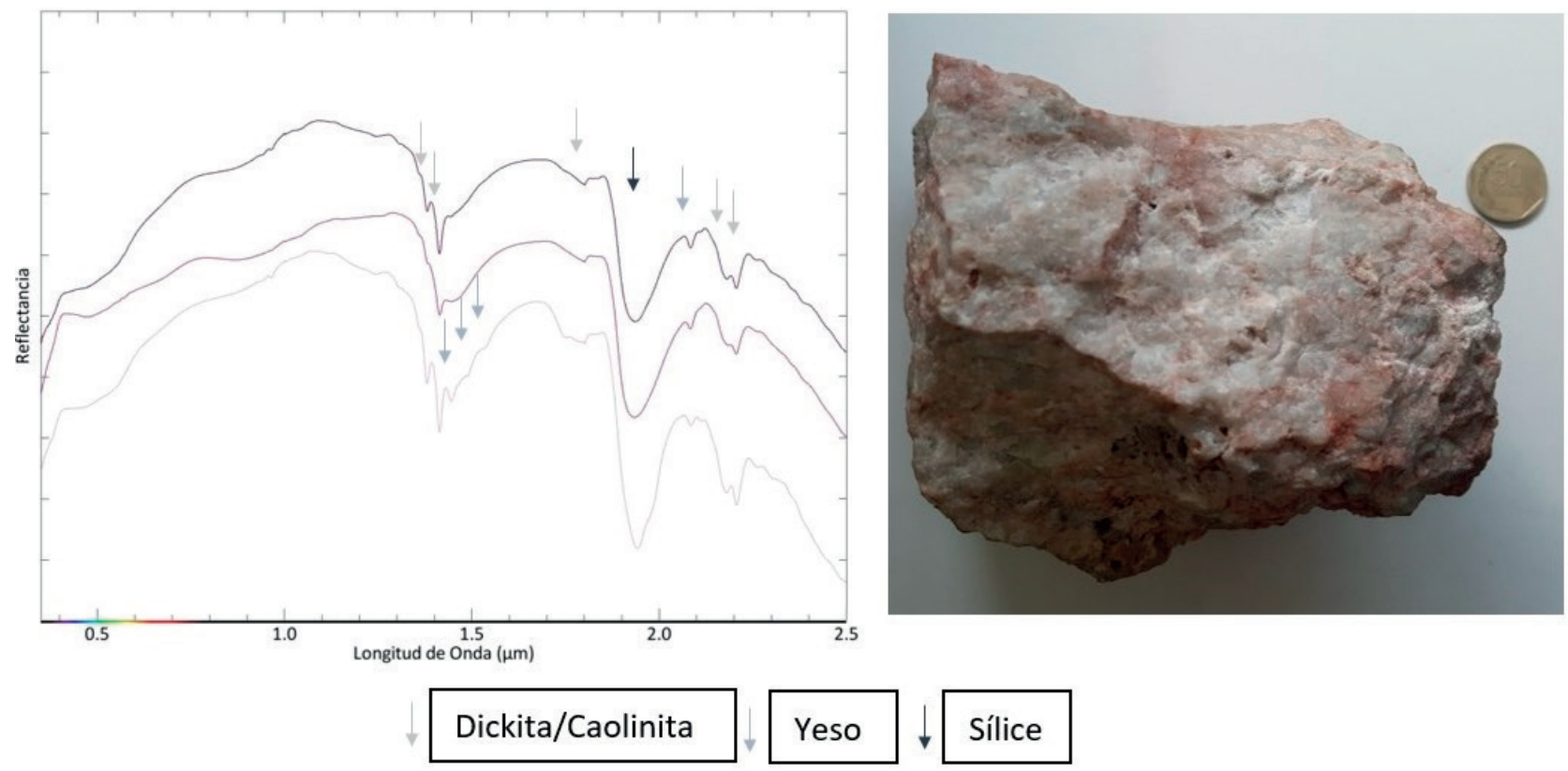

Figura 4. Gráfico con las firmas espectrales de la muestra de estructura vetiforme

El estudio de las inclusiones fluidas se realizó en los cuarzos (específicamente cuarzo II) presentando mayormente inclusiones fluidas bifásicas. El resultado de la microtermometría en las inclusiones bifásicas (Figura 5) presenta temperaturas de homogenización que van de 138 a $329^{\circ} \mathrm{C}$, con porcentajes de salinidad que varían de 0.70 a $8.95 \%$ Equivalente en peso de $\mathrm{NaCl}$.

Cabe mencionar que en una muestra estudiada proveniente de la zona $\mathrm{C}$-1, se encontraron inclusiones fluidas trifásicas con temperaturas de fusión de hielo con un mínimo de $-5.8^{\circ} \mathrm{C}$ y un máximo de $-0.4^{\circ} \mathrm{C}$, temperaturas de homogenización que están entre $560 \mathrm{a} 605^{\circ} \mathrm{C}$, y porcentajes de salinidad que van desde 44.82 a $47.66 \%$ Equivalente en peso de $\mathrm{NaCl}$.
Graficando estos resultados en el diagrama de Wilkinson (2001) para temperaturas de homogeneizaciónsalinidad se observa que puede existir una probable relación de dichas vetas epitermales con un Pórfido (Figura 6).

La alteración predominante ligada a las estructuras vetiformes es alteracion argílica (Figura 4) compuesta por caolinita-dickita-illita con \pm sericita ( $\mathrm{pH}$ acido) y en algunas la existencia de alunita y jarosita (superficial?), asociados a la silice indicando un ambiente donde las condiciones de $\mathrm{pH}$ fueron acidas con presencia de H2SO4. Por otro lado, cabe mencionar que en la roca huesped de las vetas, se han observado y determinado minerales de condiciones mas neutras como la clorita, sericita y un zona de alteración silicea. 


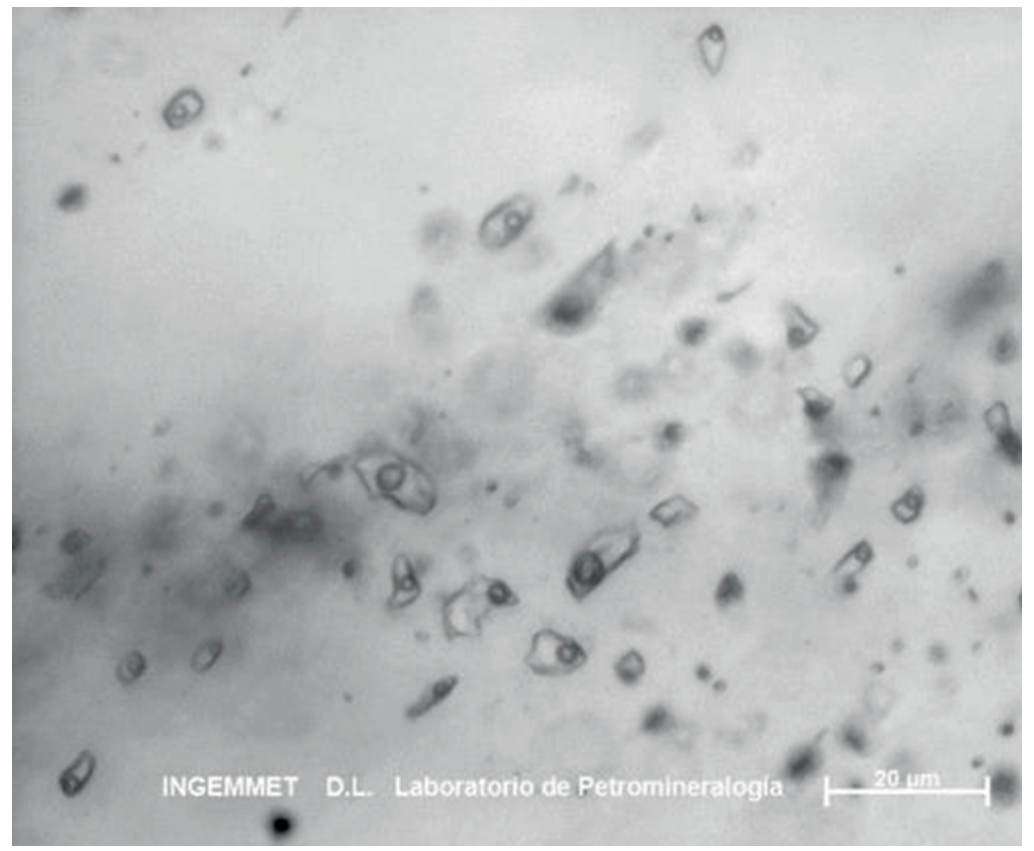

Figura 5. Familia de IFs bifásicas primarias irregulares y tabulares ricas en líquido, además de familia IFs bifásicas secundarias

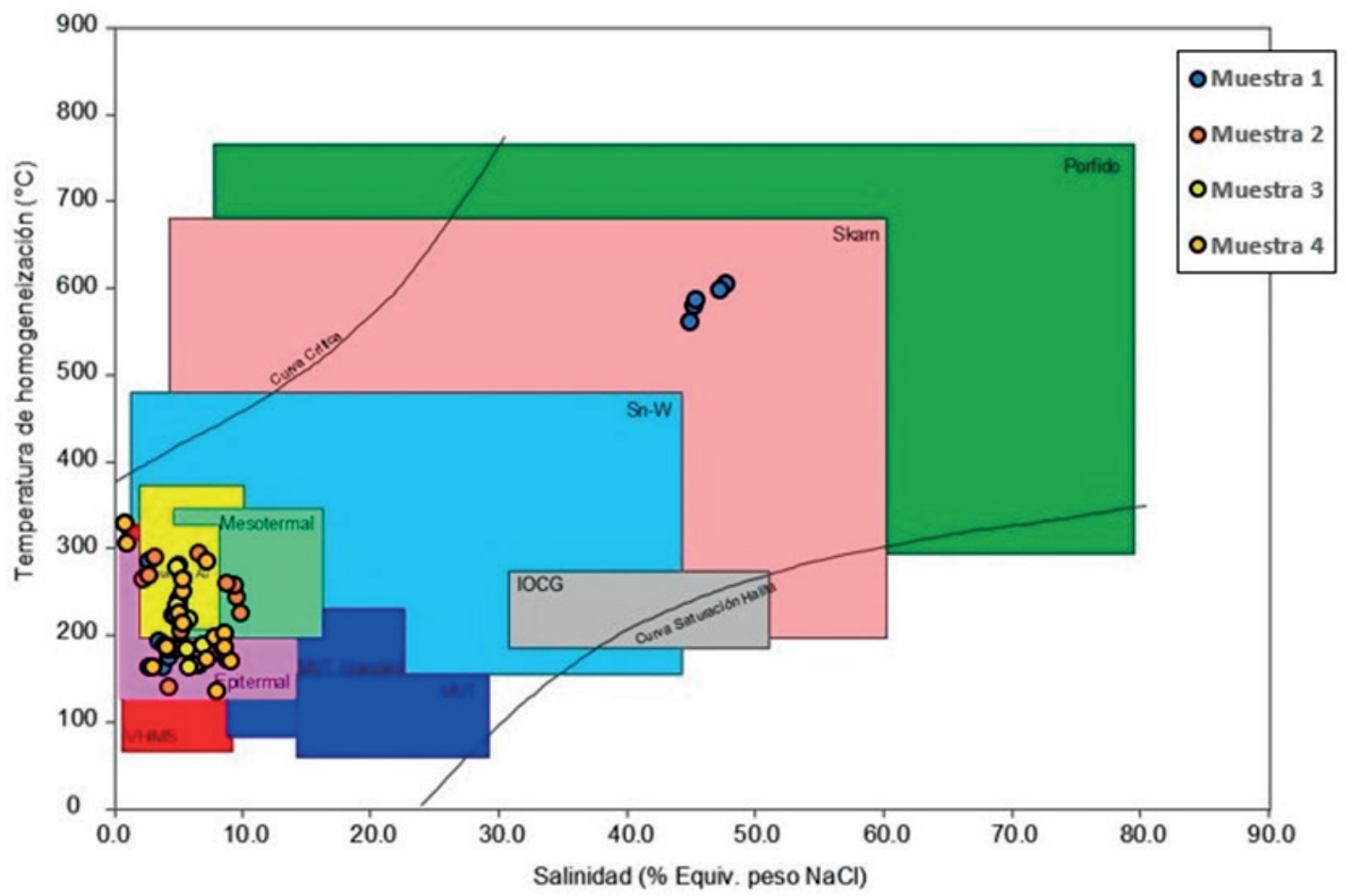

Figura 6. Diagrama de Salinidad (\%Eq. Peso $\mathrm{NaCl}$ ) vs $\mathrm{T}^{\circ}$ Homogeneización para Inclusiones Fluidas según Wilkinson (2001)

De los resultados, podemos interpretar una zona de sílice-arcilla-sericita (alteración hidrotermal más "acida") que pasa a clorita-sericita (alteración hidrotermal más "neutra") siendo sobreimpuesto por una alteración exógena de hematita-goethita. Este halo podría corresponder al del IOCG ubicado en la zona C-2 donde existen anomalías de tierras raras y posibles “Quartz Pod” (Lobo-Guerrero, 2004).

\section{DISCUSIÓN}

En las vetas epitermales (zona C-1), se ha evidenciado la existencia de varias fases hidrotermales donde la mineralización de calcopirita \pm pirrotina se relacionaría a una fase de sulfuración intermedia. Por otro lado, la alteración "acida" concentrada en las estructuras tabulares 
con una asociación mineralógica de sílice-arcilla-sericita, también contiene trazas de alunita y pirofilita, cambiando lateralmente a una zona de alteración compuesta por cuarzo-hematita-goethita-sericita-clorita. De acuerdo con lo expuesto por otros autores (Hitzman et al., 1992; Tornos et al., 2005); esto indica que dicha zona de alteración hidrotermal "ácida" presenta menor temperatura, y además según Richards \& Mumin (2013), con un pH que varía de ácida a neutro siendo una argílica relacionada con las estructuras-vetas, lo que se corrobora en la zona C-1.

Desde el punto de vista de las correlaciones de elementos y anomalías geoquímicas de mayor interés en el área de estudio indicaría la presencia de $\mathrm{Au}, \mathrm{Cu}$ y $\mathrm{Fe}$; además de elementos asociados como As, Zn, N y Co en rocas, asemejándose al registro reportados en muchos depósitos de tipo IOCG ubicados en el sur de nuestro país y al norte de Chile (Sillitoe, 2003).

Según las relaciones de campo observadas, las evidencias reportadas por los análisis de laboratorio, estudios de microscopia e inclusiones fluidas, consideramos que puede existir una conexión del sistema pórfidoepitermal y el posible yacimiento tipo IOCG oculto, como refiere Sillitoe (2003), donde menciona la existencia de pequeños yacimientos tipo pórfido en las franjas metalogenéticas de los yacimientos tipo IOCG. Por otro lado, existen evidencias de IOCG en la zona norte del Perú que han sido anteriormente publicadas por Rodríguez et al. (2011) y Biminchumo Sagástegui (2013).

Dada las evidencias mencionadas, podemos concluir que las vetas se encuentran relacionadas con la presencia de rocas intrusivas, las cuales podrían ser parte de un pórfido oculto de donde vinieron los fluidos hidrotermales que formaron las vetas epitermales de sulfuración intermedia de la zona C-1. Por otro lado, en la zona C-2, las vetas epitermales están compuestas por óxidos secundarios de hierro rellenando cavidades (llegando hasta un $45 \%$ de Fe) y siendo escasas en sulfuros. La cantidad de óxidos que son mayormente cavidades rellenas con hematita-goethita, podría estar relacionada con la parte más somera y oxidada de un yacimiento tipo IOCG profundo (en la parte norte de la zona de estudio).

\section{CONCLUSIONES}

La mineralización presente en estas vetas indica tener múltiples estadios hidrotermales de $\mathrm{qz}-\mathrm{py} \pm \mathrm{cpy} \pm \mathrm{po}$ (con 3 generaciones de qz), halos de alteración argílica con una asociación mineralógica de sílice-arcillas (caolínita/ dickita-illita)-sericita, poseendo trazas de alunita- pirofilita, gradando lateralmente a un zona de alteración compuesto por cuarzo- hematita- goethita-sericita-clorita (relacionado con evidencias superficiales del yacimiento tipo IOCG oculto probablemente ubicado dentro de zona $\mathrm{C}-2$ ).

Los resultados de microtermometría en inclusiones fluidas bifásicas de las vetas muestran temperaturas de homogenización que varían de $138^{\circ}$ a $329^{\circ} \mathrm{C}$, mientras que los rangos de salinidad varían de 0.70 a $8.95 \%$ Equivalente en peso de $\mathrm{NaCl}$. Cabe mencionar que en una muestra estudiada proveniente de la zona $\mathrm{C}-1$, se encontraron inclusiones fluidas trifásicas con temperaturas de fusión de hielo con un mínimo de $-5.8{ }^{\circ} \mathrm{C}$ y un máximo de $-0.4^{\circ} \mathrm{C}$, temperaturas de homogenización que están entre 560 a $605^{\circ} \mathrm{C}$, y porcentajes de salinidad que van desde 44.82 a $47.66 \%$ Equivalente en peso de $\mathrm{NaCl}$.

Dada a las evidencias encontradas, las vetas epitermales de sulfuración intermedia estarían relacionadas con un pórfido oculto dentro de la zona $\mathrm{C}$-1, entre tanto, en la zona $\mathrm{C}-2$ se propone la existencia de un posible depósito tipo IOCG profundo (Cerrón, J. \& Torres, A., unpubl.), donde existiría relación probable en profundidad entre el pórfido y el IOCG.

Sillitoe (2003) propone que existirían yacimientos tipo pórfidos pequeños ligados a vetas epitermales aflorantes dentro de la Franja de IOCG al norte del Perú. Así mismo, debido a la limitada información obtenida del posible IOCG oculto dentro de la zona C-2 se debería realizar más estudios y a mayor escala enfocados en corroborar la existencia del IOCG oculto, y posteriormente algunos estudios de geocronología con el fin de definir la relación genética entre ambas zonas propuestas.

\section{AGRADECIMIENTOS}

Brindamos nuestro agradecimiento por el apoyo recibido de las autoridades de la E.A.P. de Ingeniería Geológica de la Universidad Nacional Mayor de San Marcos; al equipo de los laboratorios de microscopia y teledetección del INGEMMET; al técnico en geología Raymundo Leguía, así como, a los bachilleres Yuder Espíritu, Gustavo Miranda y Sebastián Loayza.

\section{REFERENCIAS}

Biminchumo Sagástegui, E. M. G. (2013). Análisis geológico del cuerpo de "Cuarzo Pod" Caseli del IOCG DVIMIN, usando como herramientas: magnetometría, espectrometría y polarización inducida [Universidad Nacional de Ingeniería]. https://alicia.concytec.gob.pe/vufind/Record/ UUNI_3cc53f7a5dab2e422ff43194e214de38

Cobbing, E. J. (1973). IMGEMMET BOLETIN NO. A26: Geología de los cuadrángulos de Barranca, Ámbar, Oyón, Huacho, Huaral y Canta 22-h, 22-i, 22-j, 23-h, 23-i, 23-j. In IMGEMMET REPORTS. https://repositorio.ingemmet.gob. pe/handle/20.500.12544/144

Cueva, E., Mamani, M., \& Rodriguez, R. (2010). Magmatismo y geoquímica del volcanismo Albiano- Cenomaniano (Grupo Casma) y Maastrichtiano-Daniano entre Pucusana y Chimbote. XV Congreso Peruano de Geología, Resúmenes Extendidos. Sociedad Geológica de Perú, Publ. Esp., 9, 921-924. https://app.ingemmet.gob.pe/biblioteca/pdf/ CPG15-214.pdf

Guevara, C. (1980). El Grupo Casma del Perú central entre Trujillo y Mala. Sol. Soc. Geol. Perú, 67. https://www.sgp. org.pe/category/bibliovirtual/?result=4101

Hitzman, M. W., Oreskes, N., \& Einaudi, M. T. (1992). Geological characteristics and tectonic setting of proterozoic iron oxide (CuUAuREE) deposits. Precambrian Research, 58(1-4), 241-287. https://doi.org/10.1016/0301-9268(92)90121-4 
Jacay, J. (2005). Análisis de la sedimentación del cretáceo de los Andes del Perú Central. Revista Del Instituto de Investigación de La Facultad de Ingeniería Geológica, Minera, Metalurgica y Geográfica, 8(15), 49-59. https:// revistasinvestigacion.unmsm.edu.pe/index.php/iigeo/ article/view/719/571

Jacay, J., \& Sempere, T. (2005). Emplacement levels of the Coastal Batholith in Central Peru. 6th International Symposium on Andean Geodynamics (ISAG 2005, Barcelona), 1, 397-399. https://horizon.documentation.ird.fr/exl-doc/pleins_textes/ divers09-03/010040258.pdf

Lobo-Guerrero, A. (2004). Quartz "Pods", An exploration guide to iron oxide-copper-gold mineralization. SEG, 2050. https:// es.scribd.com/document/328792063/Quartz-Pods-2004

Mamani, M., Navarro, P., Carlotto, V., Acosta, H., Rodriguez, J., Jaimes, F., Santos, A., Rodríguez, R., Chavez, L., Cueva, E., \& Cereceda, C. (2010). Arcos Magmáticos MesoCenozoicos del Perú. XV Congreso Peruano de Geología. Resúmenes Extendidos. Sociedad Geológica Del Perú, Pub. Esp. $\quad N^{\circ}$ 9. https://app.ingemmet.gob.pe/biblioteca/pdf/ CPG15-135.pdf

Mamani, M., Wörner, G., \& Sempere, T. (2010). Geochemical variations in igneous rocks of the Central Andean orocline $\left(13^{\circ} \mathrm{S}\right.$ to $\left.18^{\circ} \mathrm{S}\right)$ : Tracing crustal thickening and magma generation through time and space. Bulletin of the Geological Society of America, 122(1-2), 162-182. https:// doi.org/10.1130/B26538.1

Pitcher, W. S. (1979). Algunos aspectos estructurales del Batolito costanero del Perú. INGEMMET Serie D: Estudios Regionales; $\mathrm{N}^{\circ}$ 7. https://repositorio.ingemmet.gob.pe/ handle/20.500.12544/324

Richards, J. P., \& Mumin, A. H. (2013). Magmatic-hydrothermal processes within an evolving Earth: Iron oxide-copper-gold and porphyry $\mathrm{Cu} \pm \mathrm{Mo} \pm \mathrm{Au}$ deposits. Geology, 41(7), 767770. https://doi.org/10.1130/G34275.1
Rodríguez, I., Villareal, E., Acosta, J., \& Chirif, H. (2011). Informe Geoeconómico de la región Ancash. INGEMMETDirección de Recursos Minerales y Energéticos. https://cdn. www.gob.pe/uploads/document/file/1393315/Memoria de Geología Económica de la Región Ancash.pdf

Sillitoe, R. H. (2003). Iron oxide-copper-gold deposits: An Andean view. Mineralium Deposita, 38(7), 787-812. https:// doi.org/10.1007/s00126-003-0379-7

Soler, P. (1990). Cronología y distribución espacial del magmatismo en el Peru Central durante el Cretacico Superior y el Cenozoico - Relaciones con las modalidades de la subducción de la Placa Nazca (Farallon) debajo del continente Sud-Americano. Boletín de La Sociedad Geológica Del Perú, 81, 81-86. https://www.sgp.org.pe/ category/bibliovirtual/?result $=4296$

Soler, P., \& Bonhomme, M. G. (1990). Relation of magmatic activity to plate dynamics in central Peru from Late Cretaceous to present. Special Paper of the Geological Society of America, 241, 173-192. https://doi.org/10.1130/ SPE241-p173

Tornos, F., Carriedo, J., Velasco, F., \& Tomé, C. M. (2005). GEOLOGÍA Y GEOQUÍMICA DE MINERALIZACIONES EN LOS ANDES E IBERIA. c, 1-5. https://app.ingemmet. gob.pe/biblioteca/pdf/CPG14-005.pdf

Wilkinson, J. J. (2001). Fluid Inclusions in Hydrothermal Ore Deposits. In Elsevier (Ed.), Science B.V., Lithos 55 (Vol. 13, pp. 229-272). https://doi.org/10.1016/B978-0-08-0959757.01105-0 